\title{
Los contenidos del habitar rurbano: prácticas, movilidad e identidades en las ciudades intermedias del Maule, Chile
}

\section{Stefano Micheletti}

Titulado en Ciencias Forestales y Ambientales, magíster en Cooperación para el Desarrollo y Responsabilidad Social y Ambiental. Investigador del Centro de Estudios Urbano-Territoriales y académico de la Escuela de Sociología, Universidad Católica del Maule, Chile.

smicheletti@ucm.cl

\section{Felipe Saravia Cortés}

Trabajador social, magíster en Desarrollo Local y Regional, doctor en Ciencias Sociales en Estudios Territoriales. Académico del Departamento de Ciencias Sociales, Escuela de Trabajo Social, Universidad del Bío-Bío, Chile.

fsaravia@ubiobio.cl

\section{Francisco Letelier Troncoso}

Sociólogo, magíster en Sociología, doctorando en Geografía, Planificación Territorial y Medio Ambiente. Investigador del Centro de Estudios Urbano-Territoriales y académico de la Escuela de Sociología, Universidad Católica del Maule, Chile.

fletelier@ucm.cl

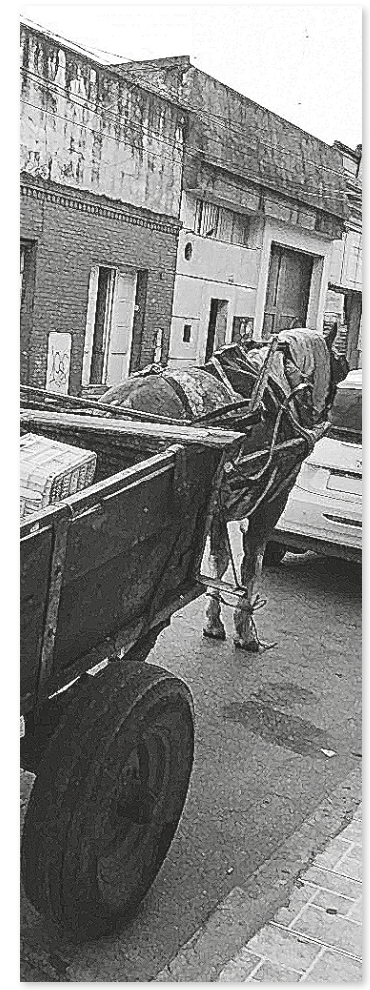




\section{Los contenidos del habitar rurbano: prácticas, movilidad e identidades en las ciudades intermedias del Maule, Chile}

\section{Resumen}

En las ciudades intermedias de la región del Maule, Chile, un alto porcentaje de personas se identifica con una adscripción identitaria un poco urbana y un poco rural. A partir de este hecho, y utilizando los datos de una encuesta aplicada a 737 personas, se desarrolla un análisis descriptivo y correlacional, orientado a identificar cuáles son las características que definen al grupo de personas que declaran sentirse "rurbanos". Se observa que si bien este grupo comparte el perfil socio-económico y laboral con el grupo que declara sentirse "urbano", se diferencia de él en tanto realiza diversas prácticas vinculadas con lo rural, presenta una mayor conexión diaria con los centros poblados menores de la región y sus miembros han nacido en menor proporción en los centros urbanos mayores. Si bien no son concluyentes, consideramos estos hallazgos como pistas para futuros trabajos, esta vez de índole más cualitativa.

\section{Palabras clave}

Rurbanidad; territorio; identidad; prácticas cotidianas; movilidad.

\section{The contents of the rurban live: practices, mobility and identities in the intermediate cities of Maule, Chile}

\begin{abstract}
In the intermediate-scale cities of the Chilean Maule Region, a number of people identify themselves as "Little bit urban-little bit rural". We aim to statistically describe and correlate this identity adscription with other key elements that characterise this group of "rurbans". We use an 737 people sample. Even though "rurbans" share socio-economic and occupational features with those who consider themselves as plain urban people, they differ regarding their rural-linked practices. "Rurbans" are also more connected with smaller urban towns in the Region, and the less of their people were born in largest cities. The evidence is not concluding, however, we consider these findings as relevant for further research, mostly for qualitative research.
\end{abstract}

\section{Keywords}

Rurbanity; territory; identity; daily practices; mobility.

\section{Os conteúdos do habitar rurbano: práticas, mobilidade e identidades nas cidades intermédias do Maule, Chile}

\section{Resumo}

Nas cidades intermediárias da região do Maule, no Chile, uma alta porcentagem de pessoas se identifica com uma atribuição de identidade um pouco urbana e um pouco rural. A partir desse fato, e utilizando os dados de uma pesquisa aplicada a 737 pessoas, é desenvolvida uma análise descritiva e correlacional, orientada para identificar quais são as características que definem o grupo de pessoas que declaram que se sentem "rurbanas". Observa-se que, embora esse grupo compartilhe o perfil socioeconômico e trabalhista com o grupo que declara sentir-se "urbano", difere dele por realizar várias práticas relacionadas ao rural, tem uma maior conexão diária com os pequenos centros populacionais da região e seus membros nasceram em menor grau nos principais centros urbanos. Embora não sejam conclusivos, consideramos essas descobertas como pistas para trabalhos futuros, desta vez de natureza mais qualitativa.

\section{Palavras chave}

Rurbanidade; território; identidade; práticas cotidianas; mobilidade. 


\section{Introducción}

Lo que se ha llamado "proceso de urbanización global" no puede ser concebido como lineal ni homogéneo, y no se concentra exclusivamente en los que tradicionalmente denominamos "centros urbanos" (BRENNER y SCHMID, 2016). En términos territoriales, no existe una sola versión de la ciudad, ni tampoco una sola manifestación de lo que conocemos como campo. La oposición rural-urbano no puede, por lo tanto, ser utilizada como si describiera contenidos estáticos, opuestos e inamovibles (CоNCHA et ÁL., 2012).

Si observamos a los habitantes de la región del Maule, en Chile, la evidencia muestra que existe una autopercepción territorial mixta: el describirse como "un poco urbano y un poco rural” es común entre la población local y no está asociado de forma lineal con las clasificaciones oficiales (propuestas por el Instituto Nacional de Estadísticas, INE). Así, por ejemplo, muchas personas que viven en ciudades se perciben como un poco urbanas y un poco rurales, o incluso como completamente rurales. En específico, investigaciones anteriores (Micheletti y Letelier, 2016; Saravia, Micheletti y Letelier, 2018) muestran tres fenómenos centrales para este territorio: primero, que en la región del Maule destacan desacoples entre las definiciones oficiales de lo que es urbano y rural y la identificación de sus habitantes. Esto evidencia que - aún en las zonas urbanas de las ciudades intermedias-hay casi un $10 \%$ de población que se percibe a sí misma como totalmente rural y que, en el sentido opuesto, parte importante de personas que viven en territorios rurales se sienten totalmente urbanas ( $15 \%$ en los pueblos de menos de 15.000 habitantes). De hecho, el $21 \%$ de quienes a nivel regional se declaran totalmente rurales vive en los cascos urbanos de ciudades intermedias, y el $32 \%$ de quienes se declaran totalmente urbanos vive en comunas que no son ciudades. En segundo lugar, la categoría de adscripción identitaria que mayor población concentra a nivel regional es "un poco urbano y un poco rural", y los cambios de adscripción intergeneracional, es decir, de padres a hijos, transitan con mayor fuerza hacia esta categoría mixta (tanto desde lo rural como desde lo urbano), y no de lo rural a lo urbano, como podría esperarse. En tercer lugar, al cruzar la variable autopercepción de ruralidad o urbanidad con la percepción que los encuestados tienen de la situación de ruralidad o urbanidad de sus padres, se encuentra que ambas variables están asociadas (lo que da cuenta de importantes cambios intergeneracionales), y que la dirección de los cambios intergeneracionales apunta una vez más hacia adscripciones territoriales subjetivas mixtas (rurbanas). 
Si bien la evidencia anterior muestra con claridad que las adscripciones mixtas son un fenómeno identitario importante, que no puede explicarse simplemente como un estado intermedio de una transición que va desde lo rural a lo urbano, es necesario comprender su contenido. ¿Tienen características comunes y diferenciadoras aquellas personas que se perciben como un poco rurales y un poco urbanas?

Nos interesa particularmente, en este sentido, analizar el fenómeno en las ciudades intermedias de la región. Para ello, el estudio indaga en las características socio-demográficas, socio-económicas, las prácticas cotidianas y de movilidad (historia residencial y movilidad cotidiana) de sujetos que se identifican como "un poco rural y un poco urbano" (rurbano, en adelante) en las ciudades intermedias del Maule, contrastándolas con las de quienes se perciben como "urbanos". Para ello, utilizamos los resultados de la "Encuesta de Caracterización Regional" realizada por el Centro de Estudios Urbano-Territoriales de la Universidad Católica del Maule, que describiremos más adelante. Junto con responder a la pregunta de investigación, se busca contribuir al debate general sobre las definiciones de lo urbano y lo rural, así como de los modelos de habitar que se asocian tradicionalmente a ambas categorías.

La investigación es de tipo exploratorio, y consideraremos las adscripciones identitarias en tres ámbitos: lo socio-ocupacional, las prácticas cotidianas y la movilidad. Entendemos que la conjunción de atributos de dichas dimensiones puede indicar la posibilidad de considerarse a sí mismo como un poco rural y un poco urbano. La especificidad del sentirse rurbano radicaría entonces en una conexión real o imaginaria con el espacio considerado tradicionalmente como rural, y que se reproduce a través de prácticas cotidianas (por ejemplo, cultivar un huerto), una trayectoria laboral y una movilidad cotidiana que se vinculan con el territorio agrario, y una historia residencial que no se limita a la ciudad, expandiéndose hacia las comunas aledañas de menor tamaño, que contienen pueblos, aldeas y caseríos.

\section{De la dicotomía rural/urbana a los enfoques integradores}

En las últimas décadas - y desde disciplinas como la Sociología, la Economía y la Geografía- se han producido nuevas aproximaciones para analizar los territorios latinoamericanos. Estas aproximaciones discuten la dicotomía urbano-rural basada en una tradición sociológica fundada en la oposición de términos como comunidad y sociedad, tradición y modernidad, agricultura e industria (Tönnies, 1986; Nisbet, 1966; Sorokin, Zimmermann y Galpin, 
1930; Germani, 1963; Solari, 1968). Desde esta tradición, la imagen de lo rural quedó fijada en términos cada vez más peyorativos- en su intrínseca "no-urbanidad" (BAIGoRRI, 1995) y su carácter agrario, tradicional, mientras que la ciudad se asoció a la modernidad, la industria, pero también a su gran tamaño y densidad poblacional, funcionalidad y complejidad de sus vínculos, y como resultado, con un modo de vida propio (WIRTH, 1938). En América Latina, esta concepción adquirió densidad sobre todo en los años posteriores a las reformas agrarias, que marcaron en algunos casos la transición a sociedades más industrializadas y menos ancladas a la lógica oligárquica tradicional en que la posesión de tierras (el latifundio) era sinónimo de poder. Se fortaleció así — cómplices también las teorías de la modernización y los aportes de autores como Rostow (1961) — la idea de que el desarrollo tenía una dirección unívoca: desde lo rural hacia lo urbano, es decir, de lo agrario a lo industrial.

La posterior implementación de un modelo socioeconómico de liberalismo radical -especialmente evidente en Chile, porque fue sustentada en la dictadura cívico-militar encabezada por Augusto Pinochet- permitió luego el despliegue de un proyecto de agroindustrialización para la exportación que significó la profundización de la migración campo-ciudad y el aumento de los flujos de intercambio entre el campo y las urbes intermediarias (productos, servicios, recursos económicos), pero también la expansión de las ciudades hacia territorios agrícolas. Todo esto terminó desdibujando límites que estaban tradicionalmente demarcados.

Paulatinamente, el debate teórico comenzó así a trasladarse hacia enfoques que ponían en duda esta visión compartimentada de los territorios, hipotetizando la existencia de "nuevas ruralidades" (PÉREz, 2001; Gómez 2002 y 2003), que tenían mayor alcance territorial y que penetraban en lo urbano gracias a la existencia de un conjunto de relaciones sociales más estrechas, propias de la condición rural. Pero este no podía ser un tema tratado solamente por los ruralistas, y diversos autores se han dedicado en la última década a reconocer una transformación de los territorios desde una perspectiva urbana. En Chile hay contribuciones importantes, entre las cuales destacan los trabajos de Berdegú, JARA, Modrego, SANCLemenTE y SchejtMAn (2010) — que proponen la idea de "Ciudades rurales" ubicadas en un punto intermedio de un gradiente de ruralidad que va desde un extremo urbano (metrópolis) hasta uno rural (caseríos) - y también de Canales y Hernández (2011) y de Canales y Canales (2012). Estos desplazan la tradicional dicotomía urbano/rural hacia el clivaje metrópolis/agrópolis, y reconocen en este último la existencia de "agrourbes" que intermedian entre lo rural y el mundo, a la vez que proveen mano de obra localizada a la agroindustria. 


\section{El habitar rurbano: aproximaciones teóricas}

Dentro de los nuevos enfoques nos interesa la perspectiva más culturalista que hace referencia a la "rurbanidad". Aquí destaca un grupo de investigadores argentinos (KeNBEL, 2006; Kenbel y Cimadevilla, 2009; Galimberti, 2011; Cimadevilla, 2014), que analizan el fenómeno desde lo identitario-productivo (las "prácticas rurbanas"). Los autores hablan de ruralización de lo urbano (o de la ciudad), entendiéndola como el proceso en que lo urbano y lo rural se entremezclan y en su síntesis postulan lo rurbano (GalimberTi, 2011). Para Cimadevilla (2014), así como se ha pensado históricamente la urbanización de la vida rural, puede concebirse el proceso contrario de ruralización de lo urbano: un proceso de interpenetración de los contrarios (GalimberTi, 2011), que en la urbe se desarrolla en la medida en que "otros modos, estilos y lógicas de reproducción no siguen los parámetros de la razón dominante y se encarnan en los actores rurales que viven y/o trabajan en la ciudad” (Kenbel y Cimadevilla, 2009, pág. 6). Esto, a través de un conjunto de prácticas "rurbanas" que han sido catalogadas según su función productiva, laboral, comercial, de uso del tiempo libre y de residencia y sociabilidad.

Es este nuestro punto de partida para plantear la idea de un "habitar rurbano", que -lejos de la idea que pretende generalizar un supuesto avance a la condición urbana, y asumiendo la existencia de una multiplicidad de condiciones de asentamientos que van desde la urbe pequeña a la metrópolis (BRENNER y SchMid, 2016)— puede representar la confluencia de diferentes procesos sociales, que se suman a la ya mencionada migración campo-ciudad:

- la existencia de una "cultura del rebusque" o bien de "actividades de refugio" (Weller, 1997) como las que llevan a cabo "carreros, cartoneros, junta basuras, etc. quienes, movilizándose en carros tirados por caballos y sin pretenderlo, modificaron los paisajes, planos, estéticas y dignidades, y también regulaciones y convivencias urbanas" (CImADEvilLa, 2014, pág. 82);

- la aparición de prácticas postmaterialistas (InGLEHART, 1971), que buscan recrear el modo de vida tradicional en el corazón de la ciudad: la agricultura urbana, el pequeño retail, la tienda de especialidad (Concha ET ÁL., 2012), todos ellos espacios y actividades posibles por el poder adquisitivo de la elite, quienes hacen de este su estilo de vida (Zukin, 1993);

- la capacidad de la ciudad latinoamericana - y chilena en este caso- de "absorber expresiones aún vívidas de lo rural o tradicional, generando espacios para su expresión ordenada y desterritorializada dentro de espacios físicos destinados a la festinación de lo urbano" (GonzÁLEZ y VIVEROS, 2007, p. 642), pero también como expresiones de resistencia y contra-racionalidad. 
De esta forma, dentro de la ciudad es posible reconocer una mixtura entre prácticas "modernas" y prácticas tradicionales, a lo que se suma el surgimiento de nuevos modos de vida que reincorporan y resignifican prácticas propias del habitar tradicional o rural (ConchA ET ÁL., 2012), sin que necesariamente se enfrente una superación o síntesis de la dicotomía (Cornejo Polar, 1990). Una forma de vida que, en definitiva, no se conecta solamente con situaciones de pobreza, marginalidad o informalidad, sino que se nutre de un sustrato más complejo de identidades y prácticas.

En el ámbito chileno, estudios anteriores demuestran la existencia de expresiones rurales en la ciudad (MicheletTi y Letelier, 2016). Concha y Letelier (2010) constatan además en términos prácticos el surgimiento de una categoría identitaria mixta ligada al proceso de hibridación de los territorios impactados por el proyecto agroindustrial en la región del Maule. Lo "rurbano" puede asociarse entonces al "acceso y al vínculo; a la libertad y a la comunidad; al anonimato y a la confianza; al pasado y al futuro, todo al mismo tiempo” (pág. 103). Esto significa la posibilidad de desvincularse de la tensión modernidad/tradición, redefiniendo lo moderno "como aquella forma de habitar el territorio que promueve la integración de valores y ventajas; lo atrasado sería lo que tiende a aislar estos valores, oponerlos o intenta anular unos en privilegio de otros” (CONCHA y Letelier, 2010, pág. 103).

Estos elementos, puestos al servicio del análisis que expondremos más adelante, nos permiten proponer la idea de la existencia de un "habitar rurbano", que suponemos sustentado en un conjunto de prácticas y condiciones de movilidad cotidiana y vinculada con la historia residencial. En términos teóricos, entendemos el "habitar rurbano" como una manera de habitar, es decir, de usar, moverse, conocer y relacionarse con el territorio, que supera la dicotomía clásica urbano-rural. Vale la pena detenerse en esta categoría, central en nuestro análisis.

El habitar excede completamente el mero ejercicio de "estar" en un lugar —normalmente una plataforma de seguridad básica que es representada por la vivienda - y relacionarse con el entorno próximo a través de actos de producción y reproducción cotidianos. Implica necesariamente un elemento de vida social, de relación con otros: la ciudad, el pueblo, el barrio como prolongación de la casa, elementos que tienen "una imagen, ritmos y características propias que se reconocen como patrimonio, un valor que tiene doble acepción: de recursos e identidad" (PigA, 2011, pág. 152). 
Saravia Madrigal (2014), analizando dos textos de Ivan Illich que tratan específicamente del habitar (La reivindicación de la casa, de 1985 y El mensaje de la choza de Gandhi, de 1978), va más lejos. Habitar significa recorrer un territorio y reconocerlo, convivirlo como acto de participación en la creación de la vida social. Es también construirlo —utilizando no solamente sus manos y sus pies, sino también máquinas y herramientas- en un ejercicio cotidiano de transformación. Es, finalmente comprenderlo, porque el territorio es esencialmente un hecho cultural.

En nuestro caso, por ser "rurbano", se trata de un habitar en el cual los límites espaciales e identitarios se difuminan tanto porque los sujetos transitan de un lugar a otro de manera cotidiana como porque re-significan los espacios más allá de la dicotomía rural/urbana. Más concretamente, planteamos inicialmente estas dimensiones del concepto de habitar rurbano como base para la comprensión del fenómeno, pero también como condicionantes de su existencia efectiva:

- autopercepción identitaria: presencia de una autopercepción mixta en términos identitarios, que involucra posibilidades de mixturas entre polos opuestos (ruralidad y urbanidad tradicionalmente entendidos);

- prácticas cotidianas: expresiones propias de la tradición rural en un contexto geográfico catalogado como urbano;

- movilidad: conexión permanente con el entorno rural de personas que habitan zonas urbanas.

\section{El caso de estudio: contexto y aspectos metodológicos del análisis}

El caso analizado es el de la región del Maule, que se encuentra emplazada en la zona central de Chile, a 200 kilómetros al sur de Santiago, y presenta una variada geografía que, desde la cordillera de los Andes, atraviesa en sentido transversal el sector de precordillera, el valle central, la cordillera de la costa y el secano, para llegar hasta la zona costera. Se le considera en general territorio rural, y presenta una matriz social y productiva ligada a lo agrario y lo campesino.

De acuerdo con los datos del Censo 2017 realizado por el Instituto Nacional de Estadísticas, alberga una población de 1.044.950 personas (INE, 2017). Talca es la capital regional, y cuenta con 220.357 habitantes; por otro lado, Curicó y Linares son las ciudades que le 
siguen en tamaño (149.136 y 93.603 habitantes, respectivamente), y son capitales de las homónimas provincias.

A nivel nacional, el Maule se ubica en los últimos lugares en los principales indicadores de desarrollo regional: pobreza, competitividad económica y productiva, ingreso y su distribución, productividad y capital humano, entre otros. Este territorio ha experimentado en los últimos 40 años grandes transformaciones, en el contexto de la implementación de un modelo de desarrollo neoliberal: por un lado, han mejorado la calidad de vida y niveles de bienestar de las personas y familias maulinas, que hoy cuentan con servicios que estaban lejos de las condiciones de vida que experimentaron sus padres; pero por otra parte, la migración hacia la ciudad se ha mantenido constante, en la medida en que la brecha en relación con las oportunidades de la ciudad aún existe: si bien vivir en sectores rurales es hoy mejor que antes, la vida en la ciudad está llena de oportunidades que quedan fuera del alcance de una familia rural. En este marco, las tres ciudades objeto de análisis (Talca, Curicó y Linares) representan nodos centrales para la región, encontrándose en el valle central y concentrando funciones vinculadas con el comercio internacional, los servicios, la oferta académica, etc.

Se analizaron los datos de una encuesta realizada cara a cara por el Centro de Estudios Urbano-Territoriales de la Universidad Católica del Maule durante el año 2014/2015 a personas mayores de 18 años, residentes en la región del Maule, y que consideró preguntas sobre percepción del territorio, prácticas cotidianas y participación social. Se utilizaron las dos submuestras que agrupan a las tres ciudades más grandes de la región y que corresponden en términos demográficos a urbes intermedias:

- residentes en la ciudad de Talca: hombres y mujeres, mayores de 18 años, que residen en el área urbana de la comuna de Talca;

- residentes en las ciudades de Curicó y Linares: hombres y mujeres, mayores de 18 años, que residan en las áreas urbanas de las comunas de Curicó y Linares.

La variable central en el análisis es la identificación urbana/rural, y obedece a la pregunta "Pensando en su vida, ¿usted siente que es una persona totalmente rural, totalmente urbana o un poco rural y un poco rural?”, que en adelante mencionamos como adscripción territorial subjetiva y asumimos como categoría "rurbana". Del total de las submuestras ( $n=823)$, se auto-identificaron como urbanos 419, y 318 como rurbanos. Se excluye a quienes se identifican como rurales $(n=86)$, debido al bajo número de encues- 
tados y al interés en este artículo en contrastar a rurbanos específicamente con urbanos. A continuación, se detalla la muestra utilizada para los análisis.

Tabla I

\begin{tabular}{lcc}
\hline Muestra utilizada & & \\
\hline Adscripción territorial subjetiva & $\mathbf{n}$ & $\%$ \\
\hline Totalmente urbana & 419 & $57 \%$ \\
\hline Un poco rural y un poco urbana & 318 & $43 \%$ \\
\hline Total & 737 & $100 \%$ \\
\hline
\end{tabular}

Fuente: elaboración propia

Se buscó determinar si existe asociación entre la adscripción territorial subjetiva y otras variables agrupadas en tres grupos: perfil socio-ocupacional, prácticas cotidianas y movilidad espacial. En el primer grupo se consideraron las variables sexo, edad, ocupación, educación, clase social autopercibida y grupo socioeconómico.

En prácticas cotidianas se utilizó la pregunta “¿me podría decir cuáles de las siguientes actividades ha desarrollado usted en el último año?”, considerando dieciséis prácticas, algunas de las cuales se asocian tradicionalmente a lo rural, como cultivar un huerto, almorzar habitualmente en casa en días de semana, hacer pan en casa, participar en fiestas religiosas, participar de una cosecha, criado de animales sin considerar mascotas domésticas, participar de fiestas tradicionales o costumbristas, y otras típicamente a lo urbano, como pedir comida a la casa, usar taxi o colectivo, ir al cine o al teatro, ir a un café o salón de té, ver un espectáculo callejero, ir al mall, comer en restaurantes, pasear por un parque, una plaza o borde de río, ir a bares o discotecas.

En cuanto a la movilidad espacial, se consideró la historia residencial, medida a través de estas variables: comuna de nacimiento, comuna de residencia hace diez años y comuna de residencia hace cinco años. Se consideró también la movilidad espacial cotidiana, a partir de las preguntas "la semana pasada, ¿usted realizó viajes a alguna otra comuna de la región?, ¿a qué comuna viajó?”, y “en los últimos 7 días, ¿realizó viajes a alguna otra comuna fuera de la región?”. 
En los cruces de cada una de estas variables con la adscripción territorial subjetiva, se aplicó coeficiente de contingencia para determinar la existencia de asociación y la intensidad de esta, que arroja un valor que fluctúa entre 0 y 1 , siendo los más cercanos a 1 aquellos con asociaciones más intensas, y los más cercanos a 0 aquellos con asociaciones débiles. Solo en aquellos casos en que se encuentra asociación estadísticamente significativa se describen las tendencias de dicha asociación.

\section{Asociación entre adscripción territorial subjetiva y perfil socioeconómico y laboral}

En términos generales, las variables analizadas en el ámbito del perfil socioeconómico y demográfico presentan una baja asociación cuando se contrastan la categoría rurbana con la urbana, tal como demuestran los datos de la tabla 2. Esto significa que el perfil es bastante homogéneo en ámbitos relevantes como distribución por sexo, edad, ocupación, educación, clase autopercibida y grupo socio-económico.

Tabla 2

\begin{tabular}{l|ccc}
\hline \multicolumn{2}{l}{ Perfil socio-económico $y$ laboral } & & \\
\hline \multirow{2}{*}{ Perfil } & Variable & $\begin{array}{c}\text { Coeficiente } \\
\text { Contingencia }\end{array}$ & Significancia \\
\hline \multirow{4}{*}{$\begin{array}{c}\text { Socio-económico } \\
\text { y laboral }\end{array}$} & Sexo & 0,059 & 0,108 \\
\cline { 2 - 4 } & Ecupación & 0,033 & 0,672 \\
\cline { 2 - 4 } & Educación & 0,090 & 0,371 \\
\cline { 2 - 4 } & Clase autopercibida & 0,026 & 0,790 \\
\cline { 2 - 4 } & Grupo socioeconómico & 0,042 & 0,867 \\
\cline { 2 - 4 } & Rama de la ocupación & 0,065 & 0,376 \\
\hline
\end{tabular}

Fuente: elaboración propia

Una mayor asociación se registra en las condiciones de habitabilidad; en este caso, y contrariamente a lo que podría esperarse al asociar lo rural con la falta de servicios básicos, los rurbanos tienen más acceso a electricidad (96 \% vs. $91 \%$ ) y alcantarillado (97 \% vs. $93 \%$ ) en sus viviendas. Otro dato interesante es que más rurbanos que urbanos tienen en el sitio donde vive su familia árboles frutales ( $39 \%$ vs. $31 \%$ ) y una huerta (10 \% vs. $5 \%$ ). 


\section{Asociación entre adscripción territorial subjetiva y prácticas cotidianas}

Para poder analizar con más propiedad la asociación entre la autopercepción y las prácticas cotidianas, clasificamos las categorías utilizadas en la encuesta en función de su relación con un imaginario tradicional rural y urbano. Aquellas que identificamos como urbanas están relacionadas con el consumo, en tanto que las prácticas que identificamos como rurales se asocian más bien al trabajo y las tradiciones culturales.

Tabla 3

\begin{tabular}{|c|c|}
\hline \multicolumn{2}{|c|}{ Prácticas asociadas a lo urbano y lo rural } \\
\hline Prácticas asociadas a lo rural & Prácticas asociadas a lo urbano \\
\hline Cultivar un huerto & Pedir comida a la casa \\
\hline \multirow{2}{*}{$\begin{array}{l}\text { Almorzar habitualmente } \\
\text { en casa en días de semana }\end{array}$} & Usar taxi o colectivo \\
\hline & Ir al cine o al teatro \\
\hline Hacer pan en casa & Ir a un café o salón de té \\
\hline Participar de una cosecha & Ver un espectáculo callejero \\
\hline \multirow{2}{*}{$\begin{array}{l}\text { Criado de animales, } \\
\text { sin considerar mascotas }\end{array}$} & Ir al mall \\
\hline & Comer en restaurantes \\
\hline \multirow{2}{*}{$\begin{array}{l}\text { Participar de fiestas } \\
\text { tradicionales o costumbristas }\end{array}$} & Pasear por un parque, una plaza o borde de río \\
\hline & Ir a bares o discotecas \\
\hline Participar en fiestas religiosas & \\
\hline
\end{tabular}

Fuente: elaboración propia

Tal como lo analizado con las variables del perfil socio-económico y laboral, se marcan algunas diferencias mínimas entre las dos categorías.

Como se aprecia en el gráfico 1, a pesar de ser estadísticamente significativas, las diferencias entre rurbanos y urbanos no sobrepasan los ocho puntos porcentuales en ninguna de las variables analizadas. En el caso de las prácticas asociadas a lo rural, los rurbanos tienen una tendencia mayor que los urbanos a participar en fiestas religiosas (diferencia de 7,5\%), 
Tabla 4

\begin{tabular}{|c|c|c|c|}
\hline \multicolumn{4}{|c|}{ Prácticas cotidianas } \\
\hline Perfil & Variable & $\begin{array}{l}\text { Coeficiente } \\
\text { Contingencia } \\
\end{array}$ & Significancia \\
\hline \multirow{16}{*}{$\begin{array}{l}\text { Prácticas } \\
\text { cotidianas }\end{array}$} & Cultivar un huerto & 0,042 & 0,260 \\
\hline & $\begin{array}{l}\text { Almorzar habitualmente } \\
\text { en casa en días de semana }\end{array}$ & 0,001 & 0,983 \\
\hline & Hacer pan en casa & 0,054 & 0,144 \\
\hline & Participar en fiestas religiosas* & 0,078 & 0,034 \\
\hline & Participar de una cosecha & 0,054 & 0,140 \\
\hline & Criado de animales, sin considerar mascotas & 0,033 & 0,365 \\
\hline & $\begin{array}{l}\text { Participar de fiestas } \\
\text { tradicionales o costumbristas** }\end{array}$ & 0,069 & 0,062 \\
\hline & Pedir comida a la casa** & 0,070 & 0,056 \\
\hline & Usar taxi o colectivo & 0,027 & 0,469 \\
\hline & Ir al cine o al teatro & 0,050 & 0,178 \\
\hline & Ir a un café o salón de té & 0,044 & 0,235 \\
\hline & Ver un espectáculo callejero & 0,024 & 0,512 \\
\hline & Ir al mall & 0,019 & 0,598 \\
\hline & Comer en restaurantes** & 0,070 & 0,058 \\
\hline & Pasear por un parque, una plaza o borde de río & 0,017 & 0,651 \\
\hline & Ir a bares o discotecas & 0,001 & 0,970 \\
\hline
\end{tabular}

Fuente: elaboración propia

$\left({ }^{*} p\right.$ valor $<0,05 ; * * p$ valor $\left.<0,1\right)$

y en fiestas costumbristas o tradicionales (diferencia de 6,9\%). En las prácticas asociadas a lo urbano, los rurbanos presentan tendencias contradictorias. Por un lado, muestran una menor tendencia a pedir comida a la casa (diferencia de 5,4\%), pero simultáneamente presentan una mayor tendencia a comer en restaurantes (diferencia de $7 \%$ ). 


\section{Asociación entre adscripción territorial subjetiva y movilidad}

En el caso de la movilidad espacial, las variables asociadas son: haber viajado durante la última semana a otra comuna de la región $(0,074)$, la comuna de nacimiento $(0,063)$ y haber viajado fuera de la región durante la última semana $(0,062)$. Ahora bien, en el caso de la comuna de nacimiento, si se excluye a quienes no nacieron en el Maule (dejando una muestra de 633 casos), el coeficiente de contingencia sube a 0,092, lo que da cuenta de que la asociación entre adscripción territorial subjetiva y lugar de nacimiento tiene una relación más intensa que con el resto de las variables.

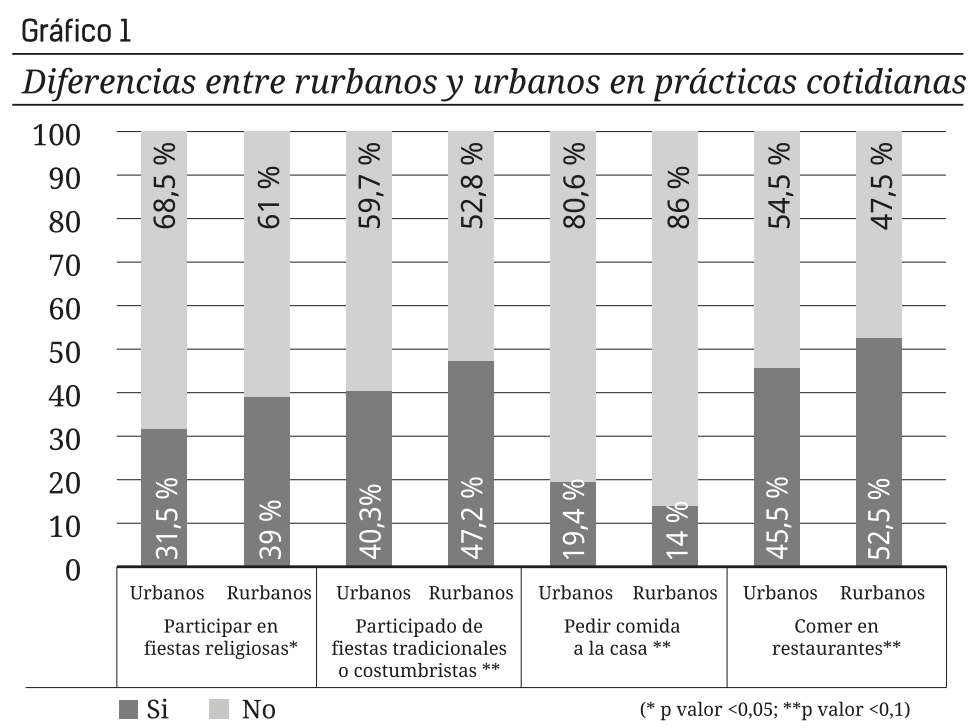

Fuente: elaboración propia

Se observa también que los rurbanos han nacido en menor proporción que los urbanos en las ciudades intermedias de la región del Maule (diferencia de 5,7 \%). En otras palabras, los sujetos con adscripción rurbana nacieron en mayor proporción que los urbanos en zonas 
Tabla 5

\begin{tabular}{|c|c|c|c|}
\hline Perfil & Variable & $\begin{array}{c}\text { Coeficiente } \\
\text { contingencia }\end{array}$ & Significancia \\
\hline \multirow{5}{*}{ Movilidad } & Comuna de nacimiento** & 0,063 & 0,086 \\
\hline & Comuna de residencia hace diez años & 0,022 & 0,547 \\
\hline & Comuna de residencia hace cinco años & 0,017 & 0,639 \\
\hline & Viaje a otra comuna de la región* & 0,074 & 0,044 \\
\hline & Viaje fuera de la región** & 0,062 & 0,092 \\
\hline
\end{tabular}

Fuente: elaboración propia

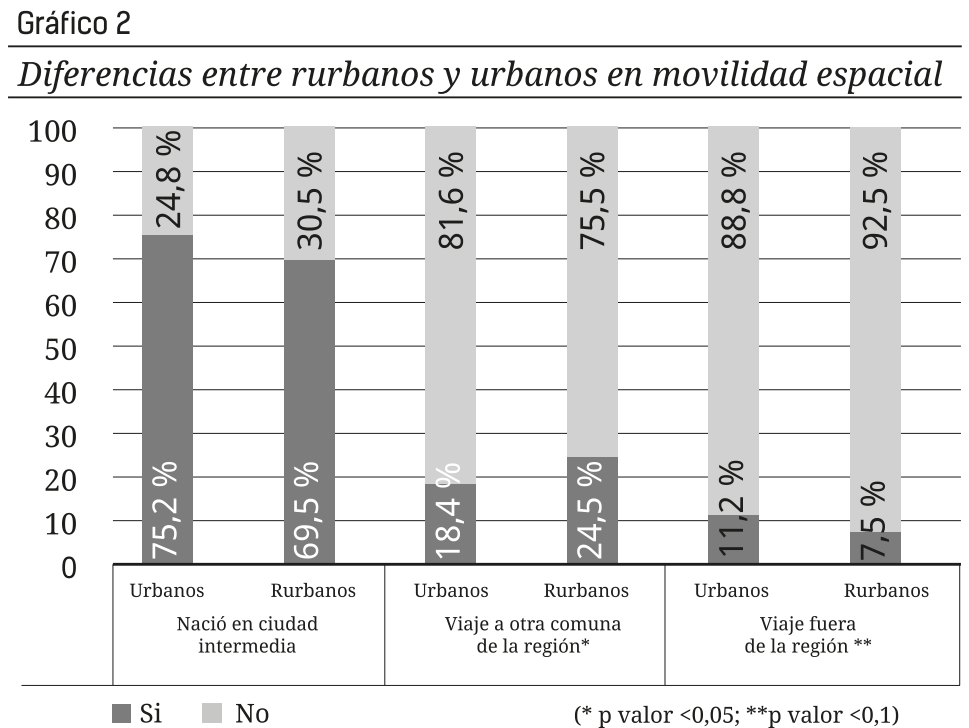

Fuente: elaboración propia 
que no son consideradas "ciudades". Esta tendencia se acentúa si se analiza solo a quienes nacieron en la región del Maule, con una diferencia del 6,8 \%.

En lo relacionado con la movilidad espacial cotidiana, se observan dos tendencias contrapuestas. Los rurbanos tienen una mayor proporción de viajes a otras comunas de la región durante la semana que los urbanos (diferencia de 6,1\%), y una menor proporción de viajes hacia fuera de la región (diferencia de 3,7\%).

\section{¿Qué hay tras la autoidentificación rururbana?}

Una primera constatación general es que ninguna de las variables consideradas en el perfil socio-ocupacional tiene diferencias significativas según adscripción territorial subjetiva, y que solo cuatro de las dieciséis prácticas consideradas y tres de las cinco variables asociadas a movilidad espacial presentan diferencias significativas. Todas son asociaciones con coeficientes de contingencia que no superan 0,080 . La baja intensidad de dichas diferencias puede estar indicando que la adscripción territorial subjetiva rurbana de quienes residen en las ciudades intermedias del Maule es un fenómeno que, si bien es claramente identificable y tiene una presencia mayoritaria en las subjetividades de los habitantes del Maule, es de difícil interpretación, y que probablemente requiera profundizaciones a través de metodologías cualitativas que ahonden en los aspectos diferenciadores aquí detectados.

En el ámbito de las prácticas emerge una mayor participación en instancias festivas tradicionales/costumbristas y religiosas; esto puede significar una mayor relación con los rituales asociados a la fe y a los ciclos productivos naturales. Pero tampoco podemos desentendernos del carácter folclórico de estos espacios, vinculados a veces con una propuesta turística estándar y promovida desde los gobiernos locales, más que con una expresión genuina, propia de las comunidades involucradas. Con todo, representan una forma distinta de construir rurbanidad, especialmente cuando en el resto de las prácticas analizadas no se marca una diferencia clara respecto de los habitantes que se perciben como completamente urbanos.

En definitiva, las prácticas que otorgan una especificidad a lo rurbano podrían ser consideradas como prácticas bisagras: participar en fiestas tradicionales o costumbristas y comer en restaurantes (piénsese especialmente en la presencia numerosa de restaurantes típicos 
o "picadas") puede ser un mecanismo mediante el cual se facilita el acceso a lo rural —sea turístico o como una especie de regreso a las raíces- por parte de quienes tienen su anclaje territorial en lo urbano.

Resulta interesante problematizar el hecho de que se encuentren diferencias asociadas a la movilidad espacial y las prácticas, pero no en atributos socio-ocupacionales. Esto puede estar indicando algo sobre la naturaleza del fenómeno en análisis. Así, el habitar rurbano no sería una forma asentada en condiciones estructurales de vida, sino en referentes culturales asociados a determinados espacios, que pueden ser móviles. Por ejemplo, quienes nacieron en territorios semirrurales o rurales de la región, pero que hoy viven en la ciudad, traen consigo -a través de un habitus incorporado - el espacio rural que se funde con lo urbano y da lugar a una autoidentificación rurbana. Es posible hipotetizar que los espacios donde transcurrió la infancia de los sujetos marcaron de manera relevante la forma en que se perciben a sí mismos, lo que no se modifica completamente con su integración a la ciudad (ya que encuentran allí un espacio de expresión), sino que más bien se hibridizan sus subjetividades.

Por otro lado, aunque las diferencias son leves, resulta notorio el contraste entre las tendencias según movilidad espacial cotidiana. Los rurbanos de las ciudades intermedias se encuentran más conectados con el hinterland de la región, pero menos conectados con el resto del país.

\section{Reflexiones finales}

En términos generales, los datos expuestos en el artículo nos permiten aportar a la idea de la obsolescencia del clivaje urbano-rural a la hora de analizar los territorios de la región del Maule. La existencia de una adscripción identitaria intermedia, que nombramos como rurbana, emerge de la encuesta aplicada por el Centro de Estudios Urbano-Territoriales, y esto contribuye a poner en tensión no solamente las ideas-fuerza contrapuestas que en los enfoques tradicionales oponían comunidad y sociedad, tradición y modernidad, rural y urbano, sino también la concepción de políticas públicas que aún se basan en esta dicotomía. En primer término, podemos entonces decir que la rurbanidad (sentirse un poco rural y un poco urbano) existe, en cuanto declaración de una porción importante de personas que habitan las ciudades intermedias del Maule. Más complejo es el análisis en relación con las formas y contenidos del habitar rurbano. 
En la exploración de las dimensiones en las que nos propusimos observar la adscripción rurbana, podemos asumir que el perfil socio-ocupacional no es un aspecto que constituya un diferenciador relevante entre urbanos y rurbanos. Sin embargo, en lo relacionado con las prácticas y la movilidad espacial existen diferencias leves aunque significativas. Lo central aquí es destacar que los elementos materiales no ayudan a entender la adscripción; mejores pistas las entregan los elementos intangibles y la movilidad. En el primer caso, es relevante la participación en espacios festivos y religiosos vinculados con lo rural, que caracteriza a los rurbanos; los datos en este caso muestran por lo demás que estos asumen a la vez de manera integral un conjunto de prácticas vinculadas con el imaginario urbano (metropolitano). En el segundo caso, las personas que se perciben un poco rurales y un poco urbanas están más conectadas cotidianamente con el territorio regional y menos con el nacional, y nacieron en menor proporción en las ciudades intermedias de la región. Sus trayectorias están marcadas, por lo tanto, por el movimiento. Es posible hipotetizar además que el acceso a espacios recreativos y religiosos rurales y la movilidad están vinculados.

De todos modos, los datos analizados no permiten definir de manera concluyente "los contenidos" de lo que definimos como "habitar rurbano", dado que las diferencias que se consideran estadísticamente significativas son en realidad bastante menores. Es este sentido, no es posible entonces afirmar que se trate de una categoría netamente definida y diferenciada de otras en sus contenidos (situación socio-económica, prácticas cotidianas, movilidad); de hecho, recoge posiblemente situaciones y trayectorias distintas, que tiene contornos poco definidos aún, y podría eventualmente interpretarse inclusive como una adscripción por negación, más que una categoría con sentido identitario fraguado: una negación de las identidades absolutas, y consecuentemente una flexibilización o relativización de estas.

Dado este escenario, se hace necesario volver a plantear preguntas e hipótesis respecto del fenómeno, interrogándose acerca de la existencia de un número tan significativo de personas que en el contexto de una ciudad intermedia se perciben un poco rurales y un poco urbanas. Pero por sobre todo, indagar en los significados asociados. Esto permitiría aclarar si la percepción mixta remite a una especie de auto-estigmatización (si asumimos, como muchos autores anteriormente mencionados proponen, que lo rural es sinónimo de atraso), o si bien existe algo en lo rural que es valorado identitariamente, y que les permite sentirse parte de algo, sin que ello implique necesariamente una forma distinta de vivir respecto de las personas que se sienten urbanas. Si fuera este el caso, podría contribuir a un mejor 
análisis la idea de comunidad perdida de José BeNGOA (2009), algo así como la memoria rural de los obreros ingleses que describe Raymond Williams en su texto Campo y Ciudad (Cit. en GonZÁLez-ParRa y Viveros, 2007).

Con todo, habría que profundizar desde la perspectiva cualitativa de investigación, sobre todo al asumir que la identidad es un proceso social complejo "en el cual los sujetos o comunidades construyen una comprensión de sí mismos, a partir de su posición en la estructura social, de los hitos que han marcado su trayectoria personal y social y de las expectativas que tienen del futuro" (Concha y Letelier, 2010, pág. 15). Y que, además, en esta época globalizada, es posible que se asocie a la búsqueda de una modernidad que se caracteriza -en palabras de Touraine - por la diversidad y multiplicación de alternativas y la capacidad de asociar pasado y porvenir (BARBERO, 1998, pág. 1).

Aun asumiendo como posible hipótesis de un nuevo trabajo que la adscripción rurbana tenga más que ver con aspectos simbólicos que concretos, no hay que descuidar los aspectos materiales del habitar que caracterizan ciudades intermedias como las que estudiamos en este artículo. El desafío en este caso se plasma en la búsqueda de las conexiones que puedan existir entre la adscripción rurbana y las prácticas rurales (laborales, recreativas, etc.) que se despliegan en lo urbano, pero también con el proceso de agroindustrialización de muchos territorios del centro-sur de Chile. Esto, como plantean CanaLES y CANALES (2012), ha llevado a una separación de las categorías "agrario" y "rural”, debido a que la actividad agraria puede realizarse hoy en día con un patrón de poblamiento no solamente rural (producción agraria primaria), sino también agro-urbano (producción agraria primaria y agroindustria), lo que sin duda alimenta el tránsito permanente entre el campo y la ciudad.

\section{Referencias bibliográficas}

BAIGORRI, Artemio (1995). De lo rural a lo urbano. Hipótesis sobre las dificultades de mantener la separación epistemológica entre Sociología Rural y Sociología Urbana en el marco del actual proceso de urbanización global. En $V$ Congreso Español de Sociología, España.

BARBERO, Jesús Martín (1998). Modernidades y destiempos latinoamericanos. En Nómadas, 8, 20-34. Bogotá. 
BENGOA, José (2009). La Identidad perdida. Identidad y cultura: desafíos de la modernización en Chile. Santiago de Chile: Ediciones Catalonia.

BERDEGUÉ, Julio, JARA, Esteban, MODREGO, Felix, SANCLEMENTE, Ximena, SCHEJTMAN, Alexander (2010). Ciudades Rurales en Chile. Documento de Trabajo 61, Programa Dinámicas Territoriales Rurales. Santiago de Chile: Rimisp.

BRENNER, Neil y SCHMID Christian (2016). La era urbana en debate. EURE, 42(127) 307-339. CANALES, Manuel y HERNÁNDEZ, María Cristina (2011). Del fundo al mundo. Cachapoal, un caso de globalización agropolitana. Espacio Abierto, 20(4), 579-605.

CANALES, Manuel y CANALES, Alejandro (2012). La Nueva Provincia: (re)poblamiento de los territorios agrarios. Chile 1982-2002. Revista Anales, Séptima Serie, 3, 155-173.

CIMADEVILLA, Gustavo (2014). Las formas de la rurbanidad - Anuncios e imágenes. En Congreso ALAIC, Pontificia Universidad Católica del Perú, Lima.

CONCHA, Claudia, ERRÁZURIZ, Tomás, LETELIER, Francisco, MICHELETTI, Stefano, RASSE, Alejandra y SALCEDO, Rodrigo (2012). ¿Urbano o rural? Repensando territorios, discursos y prácticas al margen de la metrópolis. En Congreso ALAS, Santiago de Chile.

CONCHA Claudia y LETELIER, Francisco (2009). Identidad e Identidades en el Maule, Claves para imaginar el desarrollo regional. Gobierno Regional del Maule, Talca.

CORNEJO POLAR, Antonio (1990) Una Heterogeneidad no Dialéctica: Sujeto y Discurso Migrantes en el Perú Moderno. Revista Iberoamericana. 176, 57-117.

GALIMBERTI, Silvina (2011). Rurbanidad, objetos y significaciones. Un estudio acerca de los actores rurbanos y la política pública. En XXXIV Congresso Brasileiro de Ciências da Comunicação, Recife.

GERMANI, Gino (1963). Política y Sociedad en una Época de Transición. De la sociedad tradicional a la sociedad de masas. Buenos Aires: Paidós.

GÓMEZ, Sergio (2002). La Nueva Ruralidad ¿Qué tan nueva? Valdivia: Editorial Universidad Austral de Chile.

GóMEZ, Sergio (2003). Nueva Ruralidad - Fundamentos teóricos y necesidad de avances empíricos. En Seminario internacional El mundo rural: transformaciones y perspectivas a la luz de la nueva ruralidad, Bogotá.

GONZÁLEZ-PARRA, Claudio y VIVEROS, Gustavo (2007). Pugnas por Significar: Culturas Políticas 'Rurales' No-Dialécticas. En VI Congreso Chileno de Antropología. Colegio de Antropólogos de Chile A. G, Valdivia.

INSTITUTO NACIONAL DE ESTADÍSTICAS (2017). Censo de población de Chile. 
Recuperado de: https://www.censo2017.cl/

INGLEHART, Ronald (1971). The Silent Revolution in Europe: Intergenerational Change in Post-Industrial Societies. The American Political Science Review. 65(4), 991-1017.

KENBEL, Claudia (2006). A mitad de camino entre lo urbano y lo rural: actores y actividades de rebusque. UNI revista, 22(3).

KENBEL, Claudia y CIMADEVILLA, Gustavo (2009). La rurbanidad desde el enfoque de las memorias sociales. En X Jornadas Argentinas de Estudios de Población, Asociación de Estudios de Población de la Argentina, San Fernando del Valle de Catamarca.

MICHELETTI, Stefano, y LETELIER, Francisco (2016). Aproximaciones al estudio de las prácticas rurbanas en la ciudad intermedia chilena. Actividades de rebusque en Talca. Bifurcaciones, 21.

NISBET, Robert (1967). The Sociological Tradition. Londres: Basic Books.

PIGA, José Antonio (2011). Pobladores y vivienda rural: un programa de reconstrucción después del terremoto de 1985. Revista de Geografía Espacios 1(2): 147-163.

PÉREZ, Edelmira (2001). Hacia una nueva visión de lo rural. Buenos Aires: CLACSO.

ROSTOW, Walt (1961). Las etapas del crecimiento económico: un manifiesto no comunista. Ciudad de México: Ediciones Efe.

SARAVIA, Felipe, MICHELETTI, Stefano y LETELIER, Francisco (2018). Ni urbanos, ni rurales: cambios intergeneracionales en adscripción territorial subjetiva en la región del Maule, Chile. Revista Cuaderno Urbano, 24(24), 27-46.

SARAVIA MADRIGAL, Manuel (2014). El significado de habitar. Boletín $C F+S$, 26. Valladolid, España. Recuperado de: http://habitat.aq.upm.es/boletin/n26/amsar.html

SOLARI, Aldo (1968). Sociología rural latinoamericana Buenos Aires: Paidós.

SOROKIN, Pitirim, ZIMERMANN, Carle, y GALPIN, Charles (1930). A Systematic Source Book in Rural Sociology. The University of Minnesota Press, Minneapolis.

TÖNNIES, Ferdinand (1986). El Nacimiento de mis Conceptos de "Comunidad" y "Sociedad”. Teoría Sociológica UAM Azcapozalco, 1(1), 1-5.

WELLER, Jürgen (1997). El empleo Rural no Agropecuario en el istmo Centroamericano. Revista de la Cepal, 62, 75-90.

WIRTH, Louis (1938). Urbanism as a way of life. En American Journal of Sociology, 44(1), $1-24$.

ZUKIN, Sharon (1993). Landscapes of power: From Detroit to Disneyworld. University of California Press, Oakland. 



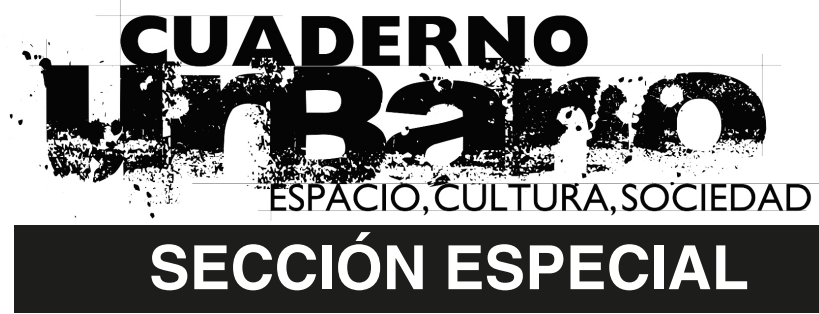


